\title{
EL MERCADO LABORAL Y LOS MÉDICOS DE FAMILIA. APORTES DESDE LA REALIDAD EN ARGENTINA
}

\author{
Ana Carolina Godoy* \\ Médica especialista en Medicina Familiar y Comunitaria, Programa SUMAR, Ministerio de Salud, Córdoba, Argentina
}

RESUMEN: El objetivo de este trabajo es identificar elementos teóricos que aportan a la investigación cualitativa sobre médicos de familia y generales (MFYG) en atención primaria de la salud (APS). En primer lugar, se describe la realidad sanitaria argentina. Varios investigadores han planteado una crisis por la escasez de MFYG disponibles para el sector público. Se han detallado aspectos como el déficit en la cantidad de ingresantes a los planes de formación y el abandono de la especialidad al cabo de algunos años. Luego se han identificado las causas. Se ha observado que los MFYG argumentan un bajo prestigio y escasos ingresos económicos como razones para el abandono de la especialidad. Finalmente, se proponen otros posicionamientos, derivados de la sociología y de la historia del sistema sanitario, que podrían enriquecer la investigación y la comprensión del fenómeno. Se concluye con la necesidad de mirar procesos colectivos para comprender el problema en contexto.

Palabras clave: Medicina familiar y comunitaria. Médicos. Atención primaria de la salud. Investigación cualitativa. Argentina.

\section{THE LABOR MARKET AND FAMILY PHYSICIANS.} CONTRIBUTIONS FROM ARGENTINA'S REALITY

ABSTRACT: The objective of this study is to identify theoretical elements that contribute to qualitative research on family and general physicians (FAGP) in primary health care. First, the Argentinean health reality is described. Several researchers have presented a crisis with a small number of FAGP available for the public sector. Aspects such as the deficit in the number of admissions to training programs and desertion of specialties after a few years have been detailed. Causes were subsequently identified. It was found that FAGP argue that low prestige and low economic income are reasons for abandoning the specialty. Finally, we propose other positions, derived from sociology and the history of health care systems that can enrich research and the understanding of this phenomenon. It was concluded that it is necessary to observe collective processes to understand the context of this problem. (Rev Mex Med Fam. 2018;5:83-8) Corresponding author: Ana Carolina Godoy, anacgodoy@yahoo.com.ar

Key words: Family and community medicine. Physicians. Primary health care. Qualitative research. Argentina.

\section{INTRODUCCIÓN}

La MFYG ha sido propuesta como especialidad clave para el desarrollo del primer nivel de atención (PNA) dentro de la estrategia de atención primaria de la salud (APS). Sin embargo, la falta de adjudicación de cargos y el abandono temprano de programas de formación es una realidad cada vez más documentada y que amenaza el cumplimiento de esos objetivos. En el análisis de esta situación se han estudiado aspectos de la formación médica, de la organización del sistema

\section{Correspondencia:}

*Ana Carolina Godoy

Obispo Marcellano, 3817, Alto Verde

C.P. 5009 Córdoba, Argentina

E-mail:anacgodoy@yahoo.com.ar 
sanitario y del mercado. Este análisis se contextualiza en el caso de Argentina, pero muchos elementos se pueden aplicar a distintos países de la región.

En el caso del sistema de salud argentino, la fragmentación se describió como una de las características principales y, además, la más problemática a la hora de analizar las dificultades en el cumplimiento del derecho a la salud de la población. La prestación de servicios se organizó en prestadores públicos, servicios de seguridad social (llamados obras sociales) y servicios privados. La responsabilidad dentro del subsector público se ha divido también de acuerdo a la organización gubernamental, quedando la responsabilidad del PNA a cargo de los municipios, con la ayuda de ciertos programas nacionales de salud. Esta fragmentación ha ido de la mano de la incidencia de intereses muy diversos que pujan dentro del campo sanitario local y que impactan, consecuentemente, en la vida laboral de los trabajadores de la salud $^{1}$. Además, la diferencia en la capacidad presupuestaria de estos efectores ha potenciado las inequidades.

Desde sus orígenes a finales de la década de 1970, la APS es un concepto que ha tenido distintos sentidos en su historia y análisis ${ }^{2}$. En primer lugar, podemos retomar el concepto de la Organización Mundial de la Salud (OMS), que la ubicó como estrategia para alcanzar un sistema de salud efectivo, es decir, que obtenga resultados en salud sostenibles para las poblaciones, abordando los determinantes sociales de la salud y llegando al nivel máximo posible de salud para cada persona $^{3}$. En este contexto, algunos autores identifican varios enfoques: a) APS selectiva, referida a limitadas intervenciones de alto impacto en salud, principalmente maternoinfantil; b) APS como puerta de entrada al sistema de salud, directamente relacionado con la disponibilidad de MFYG en este PNA; c) APS ampliada, que parte de los principios de A. Ata y hace hincapié en la idea de estrategia ${ }^{3}$.
Para reducir la morbimortalidad de la población, la APS se ha configurado como política de Estado en muchos países (incluido el nuestro). Pero estas políticas reflejan las distintas tendencias del alcance del concepto. Muchos años han pasado desde el acto fundacional en A. Ata que propuso la APS como el camino para alcanzar la «salud para todos» ${ }^{4}$, pero los modos en los que se ha buscado lograr las mejoras en salud han sido foco de debates y contradicciones $^{5}$. Algunas de estas discusiones han hecho foco en el recurso humano necesario en este nivel.

En el año 2008, la especialidad de MFYG fue definida por el Consejo Federal de Salud como una de las especialidades prioritarias en el marco de la estrategia de APS que el Ministerio Nacional y los jurisdiccionales sostienen como forma privilegiada de provisión de servicios de salud ${ }^{4}$. Por esto, ha sido estratégico ubicar el lugar laboral del MFYG como recurso clave de la APS.

Una revisión de varios estudios vinculados a la elección de MFYG concluyó que los que rechazaban la especialidad argumentaban, en primer lugar, la falta de prestigio. Otras razones, como la percepción del bajo ingreso económico y la amplitud de conocimientos también tuvieron peso a la hora de rechazar la posibilidad de especializarse en MFYG $^{6}$.

En oposición, otros estudios indicaron que los ingresos económicos no son los únicos que inciden en la elección de una especialidad. Estas contribuciones señalaron que son más importantes la capacidad de desarrollo laboral y la valoración del resultado que tendrá su trabajo para la sociedad, en oposición a beneficios más individuales ${ }^{7}$. También describieron dos perfiles de estudiantes que orientan la elección de especialidades: uno denominado perfil biocientífico, vinculado a cirugía y las superespecialidades, y otro denominado biosocial vinculado a salud pública, salud comunitaria y MFYG. Ninguno de esos grupos de estudiantes 
refirió el interés económico singular como el principal motivo de interés ${ }^{7}$.

Además de esta información, es pertinente reflexionar sobre qué aspectos del funcionamiento del sistema sanitario y laboral impactan en las posibilidades concretas de desarrollar la especialidad. El propósito de este trabajo es aportar elementos teóricos que pueden resultar útiles en la investigación sobre el capital humano en salud dentro de la estrategia de APS. Pretendemos profundizar en las características del campo laboral actual en donde se insertan los especialistas en MFYG y el modo de desarrollo de sus carreras laborales.

\section{DESARROLLO}

El crecimiento del subsector privado se ha incrementado desde la década de 1990, logrando la atracción de una importante cantidad de talento humano en detrimento del $\mathrm{PNA}^{8}$. Este fortalecimiento ocurrió en el marco de un avance de las políticas neoliberales que reconfiguraron el rol del Estado, alivianando la responsabilidad de éste para con los ciudadanos. Muchos servicios se privatizaron, y se delegaron tareas desde el rol central a instancias gubernamentales más pequeñas en lo técnico y lo presupuestario.

Si bien la capacidad de pago puede ser una razón por la cual el capital humano se sienta atraído a emplearse en el sector privado, otras razones referidas a la subjetividad que incluyen un modo de ser particular también deben ser tenidas en cuenta ${ }^{9}$. Entre estos aspectos, el trabajo en empresas de prestigio y la posibilidad de gestionar los ingresos en función de las prestaciones brindadas podrían ser valores ponderados por los que se elige el subsector privado como complemento. Algunos autores han planteado que los dos problemas del talento humano en salud son la concentración en grandes centros urbanos y la tendencia a emplearse en el sector privado $^{8}$. Afirmaron que lo que se juega no es sólo el ingreso o el modo de pago, sino un estilo de vida, que incluye, por ejemplo, vivir en ciudades con alta concentración de profesionales y tecnología, además de las características globales de la urbanidad que hacen a la vida cotidiana.

Spinelli ${ }^{10}$ propuso estudiar el concepto de campo entendido como el espacio en que confluyen actores, intereses y recursos particulares del sector. Destacó, entre otras cosas, el modo en que el trabajador se vincula con la remuneración, pero también con el proceso y el resultado de su trabajo. En ese juego vincular la realización personal o, su opuesto, la alienación son resultados posibles ${ }^{10}$. El camino del cambio en el campo de la salud no es lineal, pues hay actores con intereses que se niegan a perder hegemonía ${ }^{8}$. Pensar en los grandes sectores del sistema como actores permite analizar la dinámica del mercado más que en una simple división de funciones o financiamiento. Incluso algunos autores proponen que la lógica empresarial reinante en el mercado ha calado hondo en las subjetividades contemporáneas, hasta el punto de que cada individuo piensa en sí mismo como una empresa ${ }^{9}$.

Con respecto al sector público, los intentos de unificar el sistema no han prosperado. Los lineamientos del Banco Mundial respecto al PNA plantean una lógica de control financiero más que la restauración de la fragmentación ${ }^{8}$.

El Estado ha tenido muchas dificultades para regular las reglas del juego del mercado laboral de los médicos. La misma concepción de Estado ha cambiado desde los años de A. Ata hasta la actualidad. La profesión médica se ha caracterizado por el individualismo y la existencia de normas no escritas que regulan su actividad. Gran parte de los gestores de las instituciones de salud (tanto públicas como privadas) han sido médicos. Podemos inferir que los valores de la profesión han tenido sus traducciones en la organización del sistema de salud: gran tendencia a la hospitalización, superespecialización de la tarea, 
trabajo en lo público para fomentar el trabajo del sector privado (ya sea utilizando los recursos públicos o ganando práctica y prestigio $)^{11}$. Tanto el mercado como las sociedades científicas estimulan la necesidad de especializarse para ocupar un nicho en el mercado ${ }^{8}$.

Belmartino sostiene que, en la historia de la atención médica argentina, el reconocimiento social de la actividad de los médicos se traduce en un reconocimiento estatal ${ }^{11}$. Es decir, aquellas especialidades consideradas más prestigiosas o importantes en la sociedad tienen más beneficios por parte del Estado (en salarios, modos de contratación, etc.). Si bien se refiere al estatus de la profesión médica en comparación con otros tipos de trabajo, este análisis también puede aplicarse para ahondar en el propio campo de la medicina. De este modo, podríamos intuir que las especialidades médicas también son valoradas de distinto modo por el Estado, al igual que ocurre en el resto del campo social. Como resultado, las especialidades que más se alejan de la hiperespecialidad deberían ser las más desprestigiadas, las que presentan contratos más precarios y las de menor remuneración.

En el modelo europeo de APS, el médico generalista es a quien se responsabiliza por la autorización y coordinación de toda la atención necesaria de los usuarios ${ }^{5}$, siendo los elementos claves de la puerta de entrada al sistema. El panorama local es mucho más heterogéneo y propicia la práctica asistencial en escenarios diversos, y con recortes de la población atendida según sexo y edad ${ }^{12}$. El mercado laboral de los médicos continúa siendo un sector en el que es muy difícil sustituir el capital humano y los avances tecnológicos suelen requerir incorporación de nuevos profesionales, ubicándolos en un sector que ofrece gran autonomía para los trabajadores. A pesar de ello, los médicos no están ajenos a los cambios del mercado laboral general ${ }^{13}$.

Dos estudios realizados en nuestro país indicaron que, al cabo de unos años, los egresados de residencias de MFYG se encuentran insatisfechos con sus puestos de trabajo y no pueden ejercer plenamente la especialidad para la que se formaron ${ }^{12,14}$.

Uno de esos estudios concluyó que sería oportuno generar condiciones institucionales favorables para la permanencia en la especialidad, pero sin elementos teóricos que justifiquen esa afirmación ${ }^{14}$. Estos dos estudios han tenido limitaciones metodológicas y escaso marco teórico de análisis de los resultados obtenidos.

Si bien ubicar a los especialistas en MFYG dentro del sistema de salud es un modo clásico de vincular trabajadores y mercado laboral, otras teorías más complejas deben ser tenidas en cuenta para profundizar en la comprensión del fenómeno de estudio.

La legislación vigente en nuestro país tiene muchas herramientas que permiten abordajes integrales y humanizados en la salud. La Ley Nacional de Salud Mental, la Ley de Derechos del Paciente, la Ley de Migraciones, la Ley de Identidad de Género, entre otras, han configurado la apertura a un Estado que incluye y alcanza a las llamadas minorías. Sin embargo, estos últimos años se han vuelto a poner en discusión los alcances de algunas de estas leyes, instalando nuevamente debates públicos que parecían superados.

En la inserción laboral influyen muchos factores, además de los educativos ${ }^{13}$. En nuestro caso, pensamos que los elementos del campo de la salud operan sobre el tipo de trabajo que desarrollarán los especialistas en MFYG.

Belmartino describe el mercado de trabajo médico como tortuoso e inestable, aun después de la especialización médica ${ }^{1}$.

La inserción laboral no es un momento específico, sino el resultado de un proyecto en cierta medida lento ${ }^{15}$, y en el caso del mercado laboral de los médicos, son muchos los aspectos que influyen sobre él ${ }^{16}$.

Para el análisis de los fenómenos vinculados al mercado laboral, las teorías de la microeconomía pueden resultar de utilidad. 
En ella se estudia cómo los trabajadores y empleadores toman decisiones económicas en función de ciertas restricciones, pero también de ciertos deseos y proyecciones para el futuro ${ }^{17}$. Desde esta perspectiva, los trabajadores venden su fuerza de trabajo (renunciando a tiempo de ocio) a compradores (que buscan generar servicios de salud, en este caso). El itinerario laboral de los médicos reflejará entonces las tensiones que se juegan en esa relación de oferta y demanda.

La «nueva organización del trabajo» es un concepto que describe los mecanismos laborales que surgen a causa de la mundialización de la economía, la apertura del comercio internacional y los avances tecnológicos de las últimas tres décadas ${ }^{13}$. En ella coinciden la posibilidad de creación de empleo, pero también la disminución de los costos productivos a expensas del deterioro de las condiciones de empleo (y de vida) de los trabajadores. Entre los aspectos que afectan a esta degradación laboral hay dos de gran impacto en el mercado laboral de los médicos: la flexibilización y la precarización laboral ${ }^{13}$.

Si bien las teorías económicas descritas permiten estudiar a los trabajadores dentro de un «mercado laboral», otros conceptos del área de la sociología permiten comprender mejor el fenómeno en estudio. Ejemplo de esto es la noción de campo tal como la plantea P. Bourdieu ${ }^{18}$. En esta lógica, y con fuertes influencias del marxismo, se piensa en la sociedad como en un espacio estructurado y de conflicto en el que se manifiestan divisiones sociales. Lo que Bourdieu agrega al marxismo, nos explica Tovillas $^{18}$, es la descripción de otros capitales que permiten ser estructuradores del espacio social. En el caso de la inserción laboral de los MFYG, podemos mencionar que el capital social (es decir, el conjunto de relaciones sociales con que cuentan los individuos) puede ser influyente en los modos de acceder al empleo ${ }^{18}$. Otro aporte que realiza Bourdieu respecto a la dinámica de mercado tiene que ver con las elecciones que realizan los estudiantes sobre su futuro profesional ${ }^{19}$. A partir de algunas investigaciones, describe cómo los estudiantes de sociología de clases socioeconómicas más pobres tienden a optar por especializarse en temas más tradicionales (que les permiten una cierta estabilidad laboral), mientras que a medida que se eleva el nivel social, aumenta la frecuencia de elección de temas exóticos o de moda (que si bien puede presuponer un mayor riesgo laboral futuro, implica también una obediencia al principio de placer dentro de un marco de seguridad aprendido anteriormente). Esta conexión entre clases sociales y elecciones de temas de estudio podemos trasladarla al campo de las especialidades médicas. Este traslado es interesante para discutir cierta bibliografía que plantea el tema de la elección de MFYG como una cuestión aséptica de otras determinaciones más allá de la intención de los sujetos. Es probable, en cambio, que la división de tareas entre las especialidades médicas responda a modos de vida y necesidades de clase, quedando condicionada a necesidades materiales más o menos urgentes. Si bien puede pensarse que los profesionales médicos en general suelen tener ciertos comportamientos regulares en el mercado de trabajo, es pertinente plantear que dentro de ese conjunto existen diferencias laborales entre distintas especialidades. Y es probable que estas diferencias puedan adjudicarse, al menos en parte, a la lógica planteada por Bourdieu. Algunas especialidades (como clínica o medicina familiar) ofrecen mayor cantidad de vacantes para formación, realizan llamados frecuentes a ocupar esos cargos y tienen planes de formación de tres o cuatro años ${ }^{20}$; otras (como anestesia) tienen una fuerte limitación en el acceso de aspirantes, o tienen frecuencias de llamados menor que las otras, o exigen muchos más años de formación (con escasos ingresos). De alguna manera, esto remeda la división entre especialidades tradicionales y exóticas que utiliza Bourdieu en sus estudios, aunque sería necesario profundizar 
en el estudio de estas divisiones entre los médicos. También hay que tener en cuenta que los factores sociales ejercen una influencia, pero no siguen un determinismo mecánico ${ }^{19}$.

\section{CONCLUSIONES}

La investigación del capital humano en salud debe estar enmarcada en un análisis profundo del sistema sanitario y laboral. Más allá de las intenciones individuales de los aspirantes a residencias de MFYG, las realidades concretas operan condicionando las oportunidades del desarrollo pleno de la APS. Es por esto que los aportes de la sociología son fundamentales para avanzar en el conocimiento de este campo.

Los mecanismos de reproducción social, los efectos del neoliberalismo en la construcción de la subjetividad y la mercantilización de la medicina son los aportes que se han encontrado más relevantes.

Para llegar más efectivamente a los objetivos sanitarios propuestos es necesario pensar en los actores que serán motores del cambio. Por esto, incluir los elementos teóricos descritos puede llevar a pensar en sistemas de formación y trabajo más acordes a las necesidades subjetivas de las nuevas generaciones.

\section{BIBLIOGRAFÍA}

1. Belmartino S, Bloch C, Luppi I, Quinteros Z, Troncoso M. Mercado de trabajo y médicos de reciente graduación. Washington DC: Organización Panamericana de la Salud; 1990.

2. Silva Paim J. Desafíos para la salud colectiva en el siglo XXI. 1. ed. Buenos Aires: Lugar Editorial; 2011.

3. OPS. Renovación de la Atención Primaria de Salud en las Américas. Washington DC; 2005.

4. OMS. Declaración de Alma Ata. Kazajstán: OMS; 1978.

5. Ase I, Burijovich J. La estrategia de Atención Primaria de la Salud: ¿progresividad o regresividad en el derecho a la salud? Salud colectiva. 2009;5(1):27-47.

6. Senf $\mathrm{JH}$, Campos-Outcalt D, Kutob R. Factors Related to the Choice of Family Medicine: A Reassessment and Literature Review. J Am Board Fam Pract. 2003;16(6):502-12.

7. González García G, Madies C, Fontela M. Médicos: la salud de una profesión: entre los ideales de una vocación y los obstáculos para ejercerla. 1. ${ }^{\text {a }}$ ed. Buenos Aires: iROJO Editores; 2012.

8. Gollan D, Rovere M, Kreplak N, Kaski Fullone F, Balaña De Caro S, Kohan $\mathrm{P}$, et al. ¿Qué hacer en salud?: Fundamentos políticos para la soberanía sanitaria. Buenos Aires: Colihue; 2015.

9. Laval C, Dardot P. La nueva razón del mundo. Barcelona: Gedisa; 2013.

10. Spinelli H. Las dimensiones del campo de la salud en Argentina. Salud Colectiva. 2010;6:275-93.

11. Belmartino. La atención médica del siglo XX. Instituciones y procesos. Buenos Aires: Siglo XXI; 2005.

12. Silberman P, González MA, Godoy AC, Perrota A, Zamora C, Fernández Ponce de León R. Aprovechamiento de las becas de capacitación en Medicina Familiar y General del Ministerio de Salud de la Nación para el fortalecimiento del Primer Nivel de Atención. Un estudio descriptivo en nueve residencias de siete provincias de la Rep. Argentina. Buenos Aires; 2013.

13. Rey R. La inserción laboral de los médicos: los primeros años de ejercicio profesional y la incidencia del capital social. 2002

14. Kremer P, Daverio O, Pisani O, Nasini S, García G. Factores condicionantes de la elección y permanencia en la práctica de la medicina general y familiar como especialidad médica. Rev Argent Salud Publica. 2014;5(21):30-7.

15. Pelayo Pérez MB. Capital Social y Competencias Profesionales: Factores condicionantes para la inserción laboral. México: Edición electrónica. 2012. Disponible en http://www.eumed.net/

16. Ministerio de Salud de la Nación. Residencias del Equipo de Salud: Documento marco. Buenos Aires; 2011.

17. Pindyck RS, Rubinfeld DL. Microeconomía. Madrid: Pearson Educación, S.A.; 2009.

18. Tovillas P. Bourdieu. Una introducción. Buenos Aires: Quadratta 2010.

19. Bourdieu P, Passeron JC. Los herederos: los estudiantes y la cultura. 1. a ed. Buenos Aires: Siglo XXI editores; 2014.

20. Ministerio de Salud de la Nación. Marco de referencia para la formación en residencias médicas. Especialidad Medicina Familiar y/o General. Buenos Aires; 2010. 